Article

\title{
Queuing System with Unreliable Servers and Inhomogeneous Intensities for Analyzing the Impact of Non-Stationarity toPerformance Measures of Wireless Network under Licensed Shared Access
}

\author{
Ekaterina Markova ${ }^{1, *}$, Yacov Satin ${ }^{2}\left(\mathbb{D}\right.$, Irina Kochetkova ${ }^{1,3}{ }^{(D}$, Alexander Zeifman ${ }^{2,3,4}(\mathbb{C}$ \\ and Anna Sinitcina ${ }^{2}$ \\ 1 Applied Probability and Informatics Department, Peoples' Friendship University of Russia \\ (RUDN University), 6 Miklukho-Maklaya St, 117198 Moscow, Russia; gudkova-ia@rudn.ru \\ 2 Department of Mathematics, Vologda State University, Lenina 15, 160000 Vologda, Russia; \\ yacovi@mail.ru (Y.S.); a_zeifman@mail.ru (A.Z.); a_korotysheva@mail.ru (A.S.) \\ 3 Institute of Informatics Problems, Federal Research Center "Computer Science and Control" of the Russian \\ Academy of Sciences, Vavilova 44-2, 119333 Moscow, Russia \\ 4 Vologda Research Center RAS, Gorky Street 56A, 160014 Vologda, Russia \\ * Correspondence: markova-ev@rudn.ru
}

Received: 9 April 2020; Accepted: 12 May 2020; Published: 14 May 2020

\begin{abstract}
Given the limited frequency band resources and increasing volume of data traffic in modern multiservice networks, finding new and more efficient radio resource management (RRM) mechanisms is becoming indispensable. One of the implemented technologies to solve this problem is the licensed shared access (LSA) technology. LSA allows the spectrum that has been licensed to an owner, who has absolute priority on its utilization, to be used by other participants (i.e., tenants). Owner priority impacts negatively on the quality of service (QoS) by reducing the data bit rate and interrupting user services. In this paper, we propose a wireless multiservice network scheme model described as a queuing system with unreliable servers and a finite buffer within the LSA framework. The aim of this work is to analyze main system performance measures: blocking probability, average number of requests in queue, and average queue length depending on LSA frequencies' availability.
\end{abstract}

Keywords: LSA; queuing system; service interruption; radio resources; performance measures

\section{Introduction}

According to Cisco's latest data, the volume of mobile wireless traffic is expected to increase by almost sixty percent in the next five years, leading to a ten-fold growth of generated traffic in multiservice wireless networks [1]. In connection with that, fourth-generation (4G) wireless networks are facing a problem with the lack of radio resources necessary in the operation of multiservice network technologies such as machine-to-machine (M2M) and device-to-device (D2D) communications [2], the numbers of which are hugely increasing as the smart city vision is taking shape [3]. We remark that all communications are becoming more and more machine-oriented than humans [4].

In this context, the transition to the next fifth-generation (5G) wireless networks, the capacities of which will be higher in comparison with the current $4 \mathrm{G}$ wireless networks, seems to be the best solution to this problem. For increasing networks' capacities, researchers are investigating possible ways to extend the radio frequency range with the utilization of high radio frequency spectrum bands (i.e., over $10 \mathrm{GHz}$ ) [5] and traditional ones (i.e., under $6 \mathrm{GHz}$ ) in more efficient ways. Note that, since radio frequency spectrum bands are all allocated today, there is an urgent need to search for new RRM mechanisms. 
For this purpose, different shared access technologies to the radio frequency spectrum bands are being developed [6-9]. We will consider only one of them, namely licensed shared access (LSA) technology, which was developed by ETSI. LSA allows controlled access to spectrum shared between an owner and multiple LSA licensees (i.e., mobile operators) [10]. The owner or incumbent of the LSA band (i.e., multi-tenant band) has absolute priority over its utilization. LSA licensees can access spectrum only when the incumbent's quality of service (QoS) level is not violated. The rules governing the usage of the LSA spectrum are spelled out in a reciprocal agreement that takes into account the necessary QoS requirements. The implementation of these rules is possible through various scenarios that have a different impact on the QoS level of users served on the sharing band.

\subsection{Related Works}

According to the research conducted, restriction of interference, which is created by users of the LSA licensees in the sharing zone, is usually carried out through two main scenarios (so-called policies of interference coordination). The first is the base station (BS) power limiting (limit power policy) [11-15], and the second is the users' service interruption (shutdown policy) [16-18]. Note that the LSA licensees could have their frequency range, the so-called single-tenant band.

At the moment, the introduction of the LSA framework in wireless multiservice networks is at the development stage. Therefore, this subject is expected to attract the attention of a large number of researchers.

A review of the literature showed that the number of papers devoted to the LSA system simulation is quite large [7,19-24]. For example, in [19], theoretical considerations about various scenarios of network resource management were proposed, but analytical models were not provided. The works in $[23,24]$ offered a mechanism for distributing the LSA frequency spectrum between several LSA band tenants by using a joint auction. This scheme allowed providing unhindered access to the shared spectrum to various LSA licensees that were not related to each other. The BS of the LSA licensees was coordinated by the management organization. However, an analytical model was also not proposed.

The analytical side of this question has been poorly studied [25-28]. In [25], to obtain a model solution, cognitive radio technology was used. This technology allowed providing dynamic access to the spectrum. The evaluation of the model was carried out by analytical and simulation methods. It should be noted that the performance measures of the analytical models were investigated for stationary mode. Non-stationary mode was considered for the simulation models [29]. However, the models considered in non-stationary mode did not take into account the dependence on the periods of LSA band functioning and non-functioning on time. The methods of non-stationary mode could analyze the dependability on time and its impact on performance metrics. The first papers that examined and studied non-stationary queuing models appeared in the 1970s [30]. In recent years, such models have also been actively studied [31,32]. The research methods that we used in this work were developed in our previous studies [33,34]. This method consists of a complete study of the process $X(t)$ associated with the system and involves: (a) the construction of upper bounds for the rate of convergence to the limit regime, i.e., finding a particular moment, say $t^{*}$, starting with which, the probability characteristics of the process $X(t)$ are independent of the initial conditions (with an absolute error); (b) similar lower ratings, which are also very important and guarantee that the moment $t^{*}$ cannot be taken too small; (c) in the case of an ample state space, the construction of approximations by truncation by similar processes with fewer states and the structure of corresponding error estimates. Finally, applying the results to the system in the case of one-periodic intensities and solving the forward Kolmogorov system with the simplest initial condition corresponding to $X(0)=0$ for the truncated process on the interval $\left[t^{*}, t^{*}+1\right]$, as a result, we obtain all the main probabilistic characteristics of the process $X(t)$. We also note that only the construction of limiting characteristics, as well as only the determination of the probabilities of the process $X(t)$ are uninformative since they do not provide exhaustive information about when the initial conditions can be ignored and what happens. 


\subsection{Our Contribution}

Let us turn to the task of our paper. The combination of the LSA licensee's frequency ranges (single and multi-tenant bands) using the policies of interference coordination described above makes possible the implementation of various RRM schemes, the analysis of which allows choosing the most effective ones. In this paper, we propose a mathematical model for a radio resources access scheme in a wireless multiservice network within the LSA framework. We consider only the shared frequency spectrum (LSA or multi-tenant band). The RRM mechanism is implemented in such a way that if the incumbent needs his/her frequencies, the band becomes unavailable to the LSA license usage, while the service of tenant users is not interrupted, but goes into standby mode until the LSA band again becomes available.The missed interruptions and the usage of the buffer to serve tenant users, who are in the standby mode of LSA band recovery, are the main features and differences between the considered model and all those previously studied. The model is presented as a queuing system with unreliable servers [35] and a finite buffer and is described by the birth and death process with catastrophes [36]. In contrast to [35], adding a buffer and saving due to this current number of tenant users greatly complicate the task of studying the model. All system parameters are considered as a function of time. In accordance with the above, the main contribution in this paper is an analysis of the mobile network's model within the LSA framework, which is described as a queueing system with unreliable servers and a finite buffer operating in non-stationary mode. To research the model, the queueing theory approach was implemented $[37,38]$.

The paper is organized as follows. In Section 2, the corresponding mathematical model is constructed and the policies for efficient interference coordination are described in terms of queuing theory. Section 3 analyzes the main performance characteristics of the system-blocking probability, the average number of requests in the queue depending on the availability of the LSA band, and the bounds on the rates of convergence to the limiting characteristics of the queue. An analysis of the system transition time to stationary mode is also given. Section 4 concludes the paper by numerical examples aimed at modeling one of the LSA framework application scenarios.

\section{Mathematical Model}

Let us consider a single cell of the mobile network with an overlaid LSA framework. As a tenant of the shared frequency spectrum, we consider a mobile operator. Let the multi-tenant band have $C$ physical resource blocks (RB) and a buffer with size $r$ (i.e., $r>C$ ). We assume that requests arrive according to the Poissonprocess with rate $\lambda(t)$. For the model tractability and in order not to use approximations, we assume that service time is exponentially distributed with mean $\mu^{-1}(t)$ [39].

The radio access control (RAC) is defined in such a way that, when the user's request arrives in the system, the following outcomes are possible:

- the request always waits first in a buffer when the current number of requests in the buffer is less than $r$;

- the request's service then starts when the current number of occupied resource blocks is less than $C$;

- the request is blocked otherwise.

The incumbent, who has absolute priority over multi-tenant band utilization, uses its shared frequencies with rate $\beta(t)$ and abandons them with rate $\alpha(t)$. In relation to that, the multi-tenant band can be available or not for mobile operators. Note that, when the multi-tenant band goes into unavailable mode, servicing requests are not interrupted and return to the buffer, where they wait for the band to be operational. WE remark that all above-described rates are time-dependent.

Hereby, we simulate the model of the wireless network cell with the LSA framework as a queuing system with $C$ unreliable servers and a buffer with size $r$. Unreliable servers are characterized by ON- and OFF-period durations, which are exponentially distributed with rates $\alpha(t)$ and $\beta(t)$, respectively. All $C$ servers can go into unavailable mode (fail) only simultaneously, and conversely, 
the servers go into operational mode also simultaneously. Requests arrive according to a Poisson process with rate $\lambda(t)$, and if there are free servers, each of them occupies one server for the exponentially distributed time with parameter $\mu(t)$, while keeping its place in the queue. If all the buffer is busy upon the arrival of a request, it is blocked. Upon servers' failure, requests in service are not lost; they return to their places in the queue.

According to the above considerations, we can describe the operation of our queuing model by a Markov process $X(t)$ on the state space (Figure 1):

$$
\mathbf{X}=\{0,1, \ldots, n, \ldots, 2 r+1\}
$$

where $n=0,2, \ldots, 2 k, \ldots, 2 r$ are the states in which devices are OFF, $n=1,3, \ldots, 2 k+1, \ldots, 2 r+1$ are the states in which devices are $\mathrm{ON}$, and $k=0,1, \ldots, r$ is the number of requests in the system.
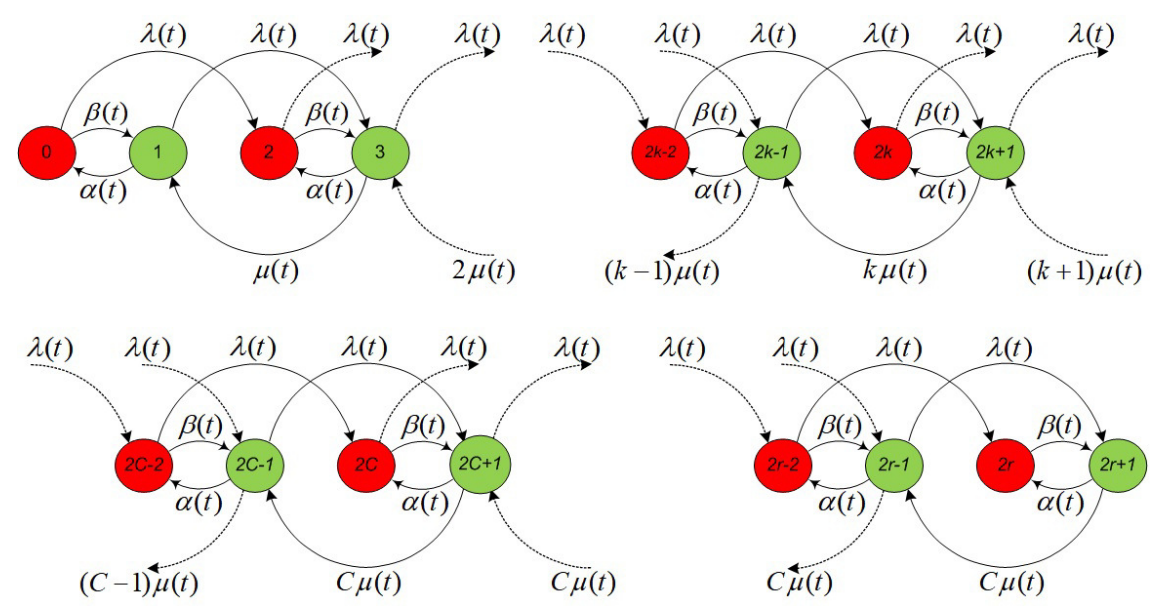

Figure 1. State transition diagram of the queuing model with catastrophes and repairs (red-devices are $\mathrm{OFF}$, green-devices are $\mathrm{ON})$.

Therefore, the elements $a\left(n, n^{\prime}\right)$ of the transposed infinitesimal generator $\mathbf{A}(t)$ are defined as follows:

$$
\begin{cases}\alpha(t), & n^{\prime}=n-1, n=2 k+1, k=0, \ldots, r \\ \beta(t), & n^{\prime}=n+1, n=2 k \\ \lambda(t), & n^{\prime}=n+2, n<2 r \\ \frac{n-1}{2} \mu(t), & n^{\prime}=n-2, n=2 k+1, k=1, \ldots, C ; \\ C \mu(t), & n^{\prime}=n-2, n=2 k+1, k=C+1, \ldots, r \\ *, & n^{\prime}=n ; \\ 0, & \text { otherwise; }\end{cases}
$$

where $*=-\left(\frac{n-1}{2} \mu(t) \cdot I(n=2 k+1, k=1, \ldots, C)+C \mu(t) \cdot I(n=2 k+1, k=C+1, \ldots, r)+\alpha(t)\right.$. $I(n=2 k+1)+\beta(t) \cdot I(n=2 k)+\lambda(t) \cdot I(n<2 r))$.

The corresponding matrix $\mathbf{A}(t)$ has a block tridiagonal form:

$$
\mathbf{A}(t)=\left[\begin{array}{ccccc}
\mathbf{N}_{0}(t) & \boldsymbol{\Lambda}_{0}(t) & \cdots & \mathbf{0} & \mathbf{0} \\
\mathbf{M}_{1}(t) & \mathbf{N}_{1}(t) & \cdots & \mathbf{0} & \mathbf{0} \\
\vdots & \vdots & \ddots & \vdots & \vdots \\
\mathbf{0} & \mathbf{0} & \cdots & \mathbf{N}_{r-1}(t) & \boldsymbol{\Lambda}_{r-1}(t) \\
\mathbf{0} & \mathbf{0} & \cdots & \mathbf{M}_{r}(t) & \mathbf{N}_{r}(t)
\end{array}\right]
$$

where: 


$$
\begin{gathered}
\mathbf{\Lambda}_{n}(t)=\left[\begin{array}{cc}
\lambda(t) & 0 \\
0 & \lambda(t)
\end{array}\right], n=\overline{0, r-1}, \\
\mathbf{M}_{n}(t)=\left[\begin{array}{cc}
0 & 0 \\
0 & n \mu(t)
\end{array}\right], n=\overline{1, C}, \\
\mathbf{M}_{n}(t)=\left[\begin{array}{cc}
0 & 0 \\
0 & C \mu(t)
\end{array}\right], n=\overline{C+1, r}, \\
\mathbf{N}_{n}(t)=\left[\begin{array}{cc}
-(\beta(t)+\lambda(t)) & \beta(t) \\
\alpha(t) & -(n \mu(t)+\alpha(t)+\lambda(t))
\end{array}\right], n=\overline{0, C}, \\
\mathbf{N}_{n}(t)=\left[\begin{array}{cc}
-(\beta(t)+\lambda(t)) & \beta(t) \\
\alpha(t) & -(C \mu(t)+\alpha(t)+\lambda(t))
\end{array}\right], n=\overline{C+1, r-1,} \\
\mathbf{N}_{n}(t)=\left[\begin{array}{cc}
-\beta(t) & \beta(t) \\
\alpha(t) & -(C \mu(t)+\alpha(t))
\end{array}\right], n=r .
\end{gathered}
$$

Let us denotethe probability that there are $n$ requests in the system (where some of them are in service, and others are waiting for a service to start or resume) $p_{n}(t)=P\{X(t)=n\}, 0 \leq n \leq 2 r+1$. It will be the probability that the continuous-time Markov chain $X(t)$ is in state $n$ at time $t$, and denote by $\mathbf{p}(t)=\left(p_{0}(t), p_{1}(t), p_{2}(t), \ldots, p_{2 r+1}(t)\right)^{T}$ the probability distribution vector at time $t$.

Then, the corresponding Kolmogorov forward equations could be written as follows:

$$
\begin{aligned}
p_{n}^{\prime}(t) & =\lambda(t) \cdot I(n>1) p_{n-2}(t)+\beta(t) \cdot I(n=2 k+1) p_{n-1}(t) \\
& +\alpha(t) \cdot I(n=2 k) p_{n+1}(t)+C \mu(t) \cdot I(n=2 k+1, k=C, \ldots, r-1) \cdot p_{n+2}(t) \\
& +\frac{n+1}{2} \mu(t) \cdot I(n=2 k+1, k=0, \ldots, C-1) p_{n+2}(t)-[\lambda(t) \cdot I(n<2 r) \\
& +\alpha(t) \cdot I(n=2 k+1)+\beta(t) \cdot I(n=2 k) \\
& +\frac{n-1}{2} \mu(t) \cdot I(n=2 k+1, k=1, \ldots, C) \\
& +C \mu(t) \cdot I(n=2 k+1, k=C+1, \ldots, r)] \cdot p_{n}(t),
\end{aligned}
$$

where $n \in \mathbf{X}, k=0, \ldots, r$.

Further, we proceed to estimate the boundaries of the rates of convergence to the limiting probability distribution.

\section{Bounds on the Rates of Convergence}

\subsection{Bounds' Defining}

Let us consider the forward Kolmogorov system (10) for the process describing the number of requests in the system.

Recall that a Markov chain $X(t)$ is called weakly ergodic, if $\left\|\mathbf{p}^{*}(t)-\mathbf{p}^{* *}(t)\right\| \rightarrow 0$ as $t \rightarrow \infty$ for any initial conditions $\mathbf{p}^{*}(0), \mathbf{p}^{* *}(0)$, where $\mathbf{p}^{*}(t)$ and $\mathbf{p}^{* *}(t)$ are the corresponding solutions of (10), where $\|\bullet\|$ denotes the $l_{1}$-norm (or distance in total variation).

The fact of the weak ergodicity of the chain $X(t)$ follows from our previous results; see the references in $[40,41]$. It is important to note that to calculate the main limiting characteristics of the process and the possibility of their practical use, we need not the fact of weak ergodicity, but the bounds of the rate of convergence. Unfortunately, the structure of the intensity matrix of the model under consideration is such that the application of the logarithmic norm cannot give us explicit estimates in the same way as in [40-42]. Therefore, it is necessary to apply less obvious research paths. Firstly, consider the logarithmic norm $\gamma$ of transposed intensity matrix $A(t)$; we have $\gamma(A(t))=$ $\max _{j}\left(a_{j j}(t)+\sum_{i \neq j} a_{i j}(t)=0\right)$ for any $t \geq 0$; hence, $\left\|\mathbf{p}^{*}(t)-\mathbf{p}^{* *}(t)\right\| \rightarrow 0$ in total variance distance decreases. Therefore, if this norm is less than $\epsilon$ for some $t_{0}$, then it is less than $\epsilon$ for any $t \geq t_{0}$. 
Secondly, since the state space (from zero to $S=2 r+1$ ) is finite and not too large, we can find the exact (at least with any practically necessary accuracy) solution of the forward Kolmogorov system with any initial condition $X(0)=i$ (which corresponds to $p(0)=e_{i}$ ) on an arbitrary finite interval $[0, l]$. Now, having found the norms of the difference between all pairs of such solutions and then choosing in the set of all solutions those for which the "extreme" initial conditions $X(0)=0$ and $X(0)=S$, we come to a value $l$ such that for $t \geq l$, the norms of the corresponding differences will be sufficiently small. Then, the solution with initial condition $X(0)=0$ gives us the periodic limiting behavior with the required accuracy on the interval $[l ; l+T]$ (and all characteristics in which one is interested).

\subsection{Performance Measures}

Let us consider the main performance measures of the system from the LSA licensee's, i.e., mobile operator's, point of view. The most interesting queuing system limiting characteristics are listed below.

- The blocking probability $B(t)$, i.e., the probability that a new user's request will be dropped:

$$
B(t)=\sum_{i=0}^{1} p_{2 r+i}(t)=p_{2 r}(t)+p_{2 r+1}(t) .
$$

- The average number of requests in the queue $Q(t)$, i.e., the number of requests awaiting in the buffer when the service will be started:

$$
Q(t)=\sum_{k=1}^{r} k p_{2 k}(t)+\sum_{k=C+1}^{r}(k-C) p_{2 k+1}(t) .
$$

- $\quad$ The average queue length, when the LSA band is operational $Q_{o n}(t)$, i.e., the number of requests waiting when the current number of occupied resource blocks will be less than $C$ :

$$
Q_{o n}(t)=\sum_{k=C+1}^{r}(k-C) p_{2 k+1}(t) .
$$

- $\quad$ The average queue length, when the LSA band is unavailable $Q_{o f f}(t)$, i.e., the number of requests waiting when devices will be $\mathrm{ON}$ and the current number of occupied resource blocks will be less than $C$ :

$$
Q_{o f f}(t)=\sum_{k=1}^{r} k p_{2 k}(t)
$$

- The resource utilization factor, $\operatorname{UTIL}(t)$, is given by:

$$
\operatorname{UTIL}(t) \cdot C=\sum_{k=1}^{r} k p_{2 k}(t)+\sum_{k=1}^{r} k p_{2 k+1}(t) .
$$

\section{Numerical Analysis}

We remark that, with the increase of the number of M2M communications due principally to smart city technology development, data traffic in modern wireless networks will grow dramatically. This technology is an example of the M2M communication paradigm in 5G networks. Due to the fact that most smart city applications are machine-oriented $[4,40]$, the interconnected sensors may become an integral part of this environment, especially in the vehicle-to-everything (V2X) scenario [41], which we consider in this section.

For the numerical analysis, we considered the case when the LSA spectrum incumbent in an urban area requires its frequency resources only occasionally, namely every 20 or $30 \mathrm{~min}$, in small and 
localized portions. In this way, we assumed that the LSA band goes into unavailable mode every 20 or $30 \mathrm{~min}$, i.e., $\alpha^{-1}=1200$ or $1800 \mathrm{~s}$. The network operator can request an underused spectrum each $20 \mathrm{~s}$, i.e., $\beta^{-1}=20 \mathrm{~s}$. Let us note that these rates do not depend on the time of the day. Furthermore, we assume that one cell of the wireless network can simultaneously serve 30 sensors, and the average duration of service is equal to $0.1 \mathrm{~s}$.

To get the values of the arrival rate $\lambda(t)$, let us consider the the source [42] (Figure 2).

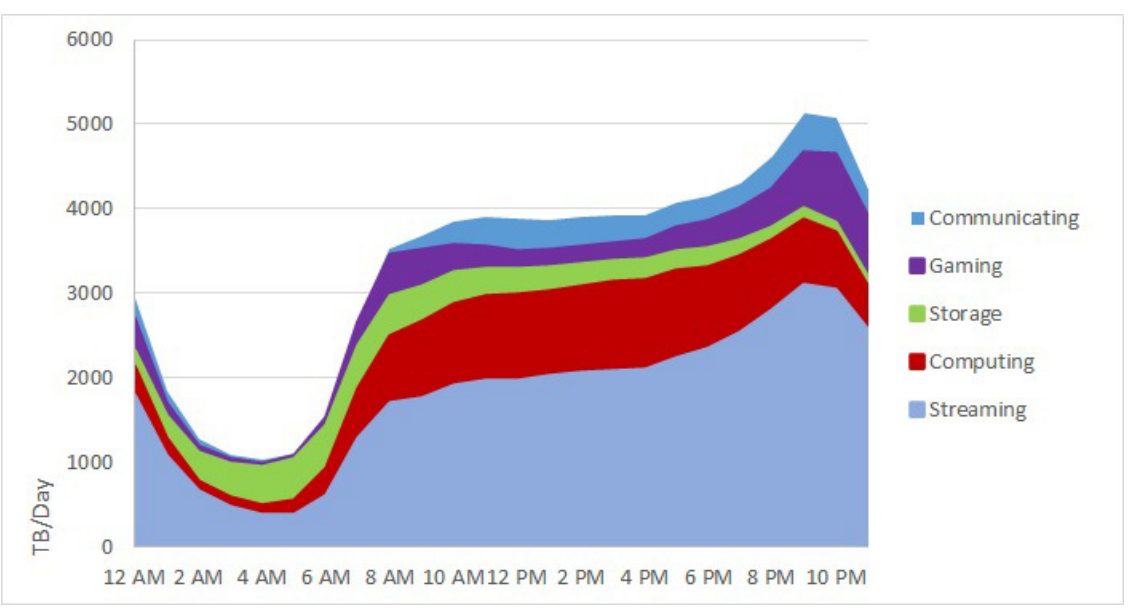

Figure 2. Application traffic daily profile in Central and Eastern Europe (TB) [42].

The data presented in Figure 2 allow us to describe the number of sensor requests for data transmission by a function of $t$. For this purpose, based on Figure 2, we can form Table 1, which shows the application traffic daily profile as a percentage.

Table 1. Application traffic daily profile in Central and Eastern Europe (\%).

\begin{tabular}{cccccc}
\hline \multirow{2}{*}{ Hours } & \multicolumn{5}{c}{ Application Traffic (\%) } \\
\cline { 2 - 6 } & Streaming & Computing & Storage & Gaming & Communicating \\
\hline 12:00 a.m. & 58.15217 & 54.97835 & 57.86164 & 57.73381 & 57.54098 \\
01:00 a.m. & 34.78261 & 33.11688 & 38.57442 & 36.33094 & 36.06557 \\
02:00 a.m. & 21.19565 & 20.12987 & 27.67296 & 25.35971 & 24.7541 \\
03:00 a.m. & 15.48913 & 15.15152 & 24.73795 & 22.30216 & 21.31148 \\
04:00 a.m. & 12.22826 & 12.55411 & 23.68973 & 21.22302 & 20 \\
05:00 a.m. & 12.5 & 14.06926 & 25.78616 & 23.20144 & 21.63934 \\
06:00 a.m. & 19.29348 & 23.80952 & 35.63941 & 32.73381 & 30.32787 \\
07:00 a.m. & 41.57609 & 48.2684 & 59.32914 & 57.19424 & 52.62295 \\
08:00 a.m. & 55.16304 & 64.50216 & 74.00419 & 74.10072 & 68.68852 \\
09:00 a.m. & 57.06522 & 68.61472 & 76.72956 & 75.35971 & 71.63934 \\
10:00 a.m. & 61.95652 & 74.24242 & 80.92243 & 76.61871 & 75.2459 \\
11:00 a.m. & 63.58696 & 76.40693 & 82.18029 & 75.89928 & 76.22951 \\
12:00 p.m. & 63.8587 & 76.83983 & 81.97065 & 74.82014 & 75.7377 \\
01:00 p.m. & 65.21739 & 77.92208 & 82.38994 & 75.17986 & 75.57377 \\
02:00 p.m. & 66.57609 & 79.43723 & 83.43816 & 76.07914 & 76.22951 \\
03:00 p.m. & 67.3913 & 80.95238 & 84.48637 & 76.97842 & 76.55738 \\
04:00 p.m. & 67.66304 & 81.38528 & 84.90566 & 77.69784 & 76.55738 \\
05:00 p.m. & 72.01087 & 84.19913 & 87.21174 & 80.7554 & 79.5082 \\
06:00 p.m. & 75.54348 & 85.28139 & 88.05031 & 82.3741 & 81.14754 \\
07:00 p.m. & 82.06522 & 88.52814 & 90.56604 & 85.61151 & 84.09836 \\
08:00 p.m. & 90.21739 & 93.72294 & 94.54927 & 90.64748 & 90.16393 \\
09:00 p.m. & 100 & 100 & 100 & 100 & 100 \\
10:00 p.m. & 98.36957 & 95.88745 & 95.59748 & 99.64029 & 99.01639 \\
11:00 p.m. & 83.42391 & 79.22078 & 79.87421 & 83.63309 & 82.29508 \\
\hline
\end{tabular}


The Fourier 2 transform can approximate the dependencies obtained in Table 1 into the function $\lambda(t)=a_{0}+a_{1} \cos (w \cdot t)+b_{1} \sin (w \cdot t)+a_{2} \cos (2 w \cdot t)+b_{2} \sin (2 w \cdot t)$. Table 2 summarizes the initial data for the considered example. Table 3 contains the coefficients that are necessary for calculating the arrival rate $\lambda(t)$.

Table 2. Main framework notations.

\begin{tabular}{cc}
\hline Notation & Value \\
\hline$C$ & 30 \\
$r$ & 50 \\
$\alpha^{-1}$ & $1200,1800 \mathrm{~s}$ \\
$\beta^{-1}$ & $20 \mathrm{~s}$ \\
$\lambda(t)$ & $a_{0}+a_{1} \cos (w \cdot t)+b_{1} \sin (w \cdot t)+a_{2} \cos (2 w \cdot t)+b_{2} \sin (2 w \cdot t)$ \\
$\mu^{-1}$ & $0.1 \mathrm{~s}$ \\
\hline
\end{tabular}

Table 3. Arrival rate $\lambda(t)$ by using traffic daily profile approximation (Fourier 2).

\begin{tabular}{cccccc}
\hline$a_{\mathbf{0}}$ & $\boldsymbol{a}_{\mathbf{1}}$ & $\boldsymbol{b}_{\mathbf{1}}$ & $\boldsymbol{a}_{\mathbf{2}}$ & $\boldsymbol{b}_{\mathbf{2}}$ & $\boldsymbol{w}$ \\
\hline \multicolumn{7}{c}{ Streaming traffic } \\
\hline 57.78 & -5.188 & -29.19 & 3.973 & -20.11 & 0.2601 \\
\hline \multicolumn{7}{c}{ Computing traffic } \\
\hline 63.72 & -13.39 & -30.77 & 3.483 & -19.45 & 0.2605 \\
\hline \multicolumn{7}{c}{ Storage traffic } \\
\hline 69.18 & -14.16 & -25.95 & 1.884 & -18.02 & 0.2605 \\
\hline \multicolumn{7}{c}{ Gaming traffic } \\
$\mathrm{v} 65.92$ & -11.25 & -24.58 & 1.679 & -20.26 & 0.2601 \\
\hline \multicolumn{7}{c}{ Communicating traffic } \\
\hline 64.71 & -11.1 & -25.62 & 2.983 & -19.66 & 0.26 \\
\hline \multicolumn{7}{c}{}
\end{tabular}

We further focused on the following set of metrics of interest: the blocking probability $B(t)$ and the average number of requests in the queue $Q(t)$, based on the arrival rate $\lambda(t)$.

For this purpose, first of all, we considered the data corresponding to the first type of traffic (streaming traffic) presented in Table 3. Next, we define a time interval (Figure 3) on which the system ceases to depend on the selected initial conditions, the first of which $X(0)=0$ corresponds to the fact that the system is empty and the second $X(0)=101$ to the fact that the system is filled.

According to Figure 3, the system ceases to depend on the selected initial conditions, starting with the value of $150 \mathrm{~s}$. From this point onwards, a complete coincidence of the curves is observed. A similar situation occurs for the case of $\alpha^{-1}=1800 \mathrm{~s}$. Note that the obtained interval is suitable for estimating the other considering the queuing system's limiting characteristics. Let us pass to an estimation of their limiting values.

Figure 4 shows that frequent LSA band transitions into unavailable mode lead to the blocking probability $B(t)$ (Figure $4 \mathrm{a}$ ) and the average queue length $Q(t)$ (Figure $4 \mathrm{~b}$ ) increasing. In particular, with $\alpha^{-1}=1200 \mathrm{~s}$, the performance measures of the system are almost one and a half times worse than for $\alpha^{-1}=1800 \mathrm{~s}$, namely the limiting values of the blocking probability and the average queue length for $\alpha^{-1}=1200$ approximately equal 0.016 and 0.82 , and for $\alpha^{-1}=1800$, they approximately equal 0.011 and 0.55 , respectively.

Furthermore, Figure 4 allows selecting a more accurate interval, suitable for analyzing the system performance measures, namely the time interval from 270 to $300 \mathrm{~s}$, at which the system goes to steady-state. We used this interval to perform a comparative analysis of the system performance measures, which worked with different types of traffic. Namely, consider the dependence of the 
blocking probability (Figure 5a) and the average queue length (Figure 5b) on the types of traffic generated in the system. Let us recall that the data for carrying out such an analysis are presented in Table 3.

According to the obtained results, the maximum blocking probability and the queue length were reached when traffic of type "storage" was transmitted. The minimal blocking probability and the queue length were reached when traffic of type "streaming" was transmitted. Note that the numerical analysis presented the results that allowed us to estimate only the average length of the queue $Q(t)$, which did not depend on the state of the LSA band. This was due to the fact that the value of $Q(t)$ practically coincided with the value of performance measure $Q_{o n}(t)$ (average queue length, when the LSA band was operational) and was slightly larger than the value of $Q_{o f f}(t)$.

This allowed us to obtain an upper bound for these three characteristics.

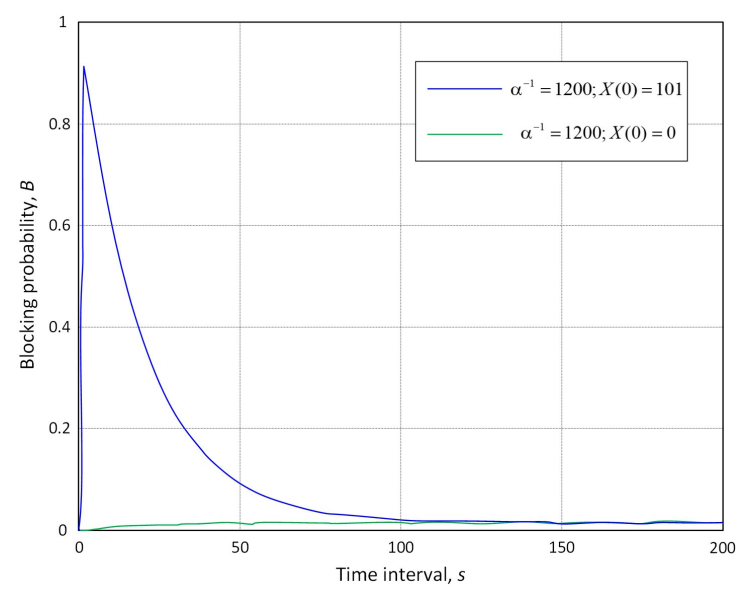

Figure 3. Estimating the time interval for blocking probability $B(t)$ analysis.

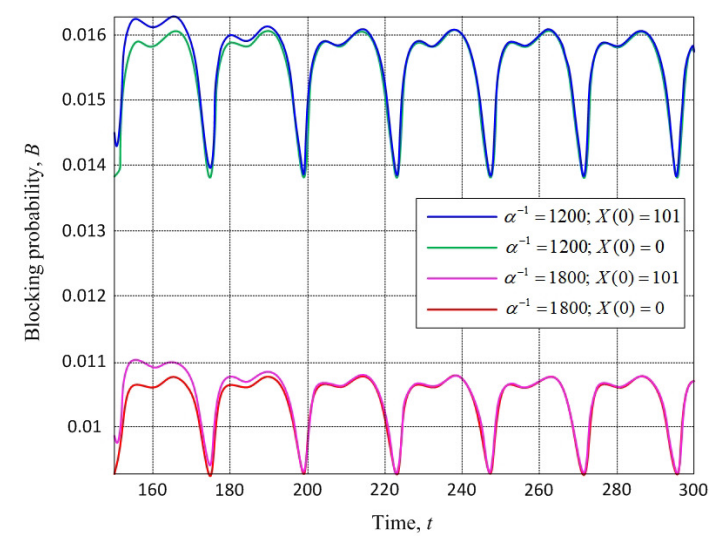

(a)

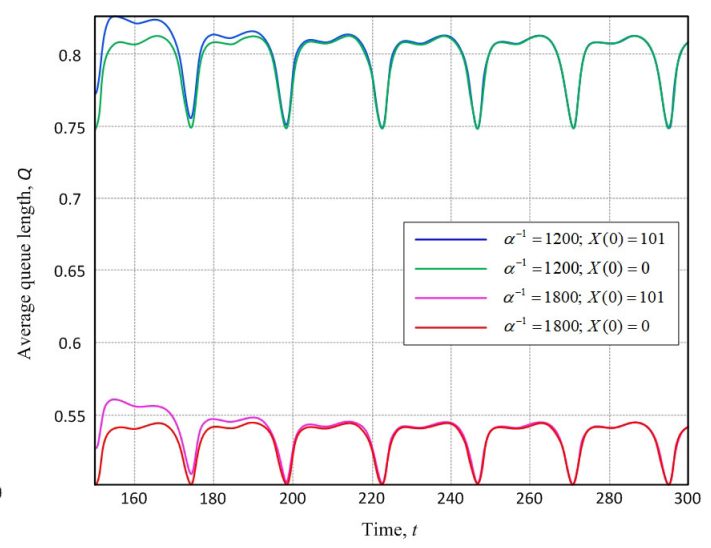

(b)

Figure 4. Estimating of limiting values for blocking probability (a) and average queue length (b). 


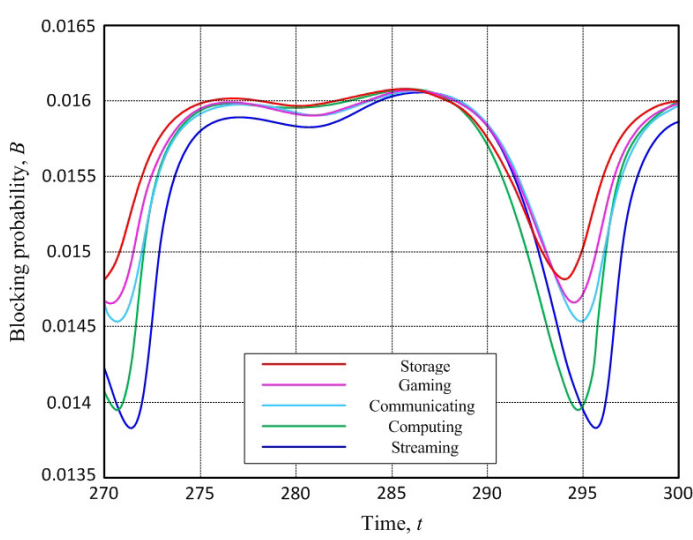

(a)

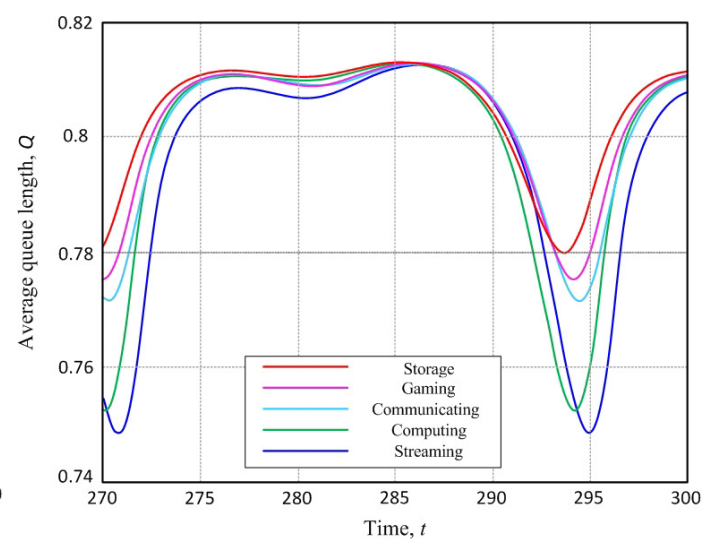

(b)

Figure 5. Blocking probability (a) and average queue length (b) depending on traffic type.

\section{Conclusions}

In the conditions of the rapid growth of data traffic volume, which is generated in modern multiservice networks, the LSA framework becomes one of the possible solutions to the frequency range shortage problems, which arises in this case. In this paper, we described a characteristic urban LSA use case, where the spectrum license owner in a smart city used its frequency resources only occasionally. We analyzed the LSA usage for the V2X scenario, within the framework of the smart city concept. For the analysis, we considered the case when the LSA licensee did not have its frequency range and he/she had access only to the LSA band. This access was modeled as a queuing system with the queue, catastrophes, and repairs. To assess the model performance measures, we analyzed the bounds on the rates of convergence to limiting characteristics: the blocking probability, the average number of requests in the queue, the average queue length, when the LSA band was operational and unavailable based on the arrival rate, which depended on the time of the day and type of generated traffic.

In this work, we considered a case of the non-stationarity of different types of rates (intensities). The corresponding mathematical method could only be applied for specific types of one-dimensional stochastic processes. For calculating delay metrics, we plan to use the apparatus of the embedded Markov chain (EMC) (as Little's law in the form $\lambda(1-B(t)) W(t) \neq Q(t)$ cannot be applied due to the service unreliability). After, a novel approach for analyzing non-stationarity, EMC should be developed. As the first step of further work, we plan to simulate the system to calculate the expected waiting time for data in the queue and total queuing delay. Since in this paper, we considered a kind of $\mathrm{M} / \mathrm{M} / \mathrm{m} / \mathrm{mqueueing}$ model to describe the RAC scheme with exponentially distributed service times, we further plan to consider a more complex type of queueing model, for example $\mathrm{G} / \mathrm{G} / \mathrm{m}$, which will make the model more realistic.

Author Contributions: Conceptualization, A.Z.; software, E.M.; investigation, E.M. (Sections 1 and 4), Y.S. (Sections 2 and 3), I.K. (Sections 2 and 3), and A.Z. (Sections 2 and 3); writing, original draft preparation, E.M., A.S., Y.S., and I.K.; writing, review and editing, A.Z. All authors read and agreed to the published version of the manuscript.

Funding: Sections 2 and 3 were written by Satin, Zeifman, and Kochetkova under the support of the Russian Science Foundation, Project 19-11-00020. The publication has been prepared with the support of the "RUDN University Program 5-100" (recipient Markova, Sections 1 and 4).

Conflicts of Interest: The authors declare no conflict of interest. 


\section{References}

1. Cisco Visual Networking Index: Global Mobile Data Traffic Forecast Update, 2017-2022 White Paper; Cisco: San Jose, CA, USA, 2019.

2. Laya, A.; Alonso, L.; Alonso-Zarate, J. Is the Random Access Channel of LTE and LTE-A Suitable for M2M Communications? A Survey of Alternatives. IEEE Commun. Surv. Tutor. 2014, 16, 4-16.

3. Ali, M.S.; Hossain, E.; Kim, D.I. LTE/LTE-A random access for massive machine-type communications in smart cities. IEEE Commun. Mag. 2017, 55, 76-83. [CrossRef]

4. Afzal, M.K.; Zikria, Y.B.; Mumtaz, S.; Rayes, A.; Al-Dulaimi, A.; Guizani, M. Unlocking 5G Spectrum Potential for Intelligent IoT: Opportunities, Challenges, and Solutions. IEEE Commun. Mag. 2018, 56, 92-93. [CrossRef]

5. ITU-R M.2083: IMT Vision-Framework and Overall Objectives of the Future Development of IMT for 2020 and Beyond; ITU: Geneva, Switzerland, 2015. [CrossRef]

6. Bajracharya, R.; Shrestha, R.; Zikria, Y.B.; Kim, S.W. LTE in the Unlicensed Spectrum: A Survey. IETE Tech. Rev. 2016, 35, 78-90. [CrossRef]

7. Onidare, S.; Navaie, K.; Ni, Q. On the Spectrum and Energy Efficiency in Dynamic Licensed Shared Access Systems: A Multiobjective Optimization Approach. IEEE Access 2019, 7, 164517-164532. [CrossRef]

8. Maule, M.; Moltchanov, D.; Kustarev, P.; Komarov, M.; Andreev, S.; Koucheryavy, Y. Delivering Fairness and QoS Guarantees for LTE/Wi-Fi Coexistence under LAA Operation. IEEE Access 2018, 6, 7359-7373.

9. Basaure, A.; Sridhar, V.; Hämmäinen, H. Adoption of dynamic spectrum access technologies: A system dynamics37approach. Telecommun. Syst. 2016, 63, 169-190. [CrossRef]

10. ETSI TS 103 379: Reconfigurable Radio Systems (RRS); Information Elements and Protocols for the Interface between LSA Controller (LC) and LSA Repository (LR) for Operation of Licensed Shared Access (LSA) in the $2300 \mathrm{MHz}-2400 \mathrm{MHz}$ Band; ETSI: Valbonne, France, 2017.

11. Adou, K.Y.; Markova, E.V.; Gudkova, I.A. Approximate Product Form Solution for Performance Analysis of Wireless Network with Dynamic Power Control Policy. Lect. Notes Comput. Sci. 2019, 11965, 379-390. [CrossRef]

12. Yu, C.-M.; Tala't, M.; Shen, L.-H.; Feng, K.-T. A Multi-objective Model Checking for Transmission Policy Optimization in Hybrid Powered Small Cell Networks. IEEE Access 2020, 8, 71339-71352. [CrossRef]

13. Yu, C.-M.; Tala't, M.; Feng, K.-T. On hybrid energy utilization for harvesting base station in $5 \mathrm{G}$ networks. Energy Sci. Eng. 2019, 8, 768-778. [CrossRef]

14. Hu, C.; Dong, Y. Age of information of two-way data exchanging systems with power-splitting. J. Commun. Netw. 2019, 21, 295-306. [CrossRef]

15. Moltafet, M.; Leinonen, M.; Codreanu, M.; Pappas, N. Power Minimization in Wireless Sensor Networks with Constrained AoI Using Stochastic Optimization. In Proceedings of the 53rd Asilomar Conference on Signals, Systems, and Computers (ACSSC 2019), Pacific Grove, CA, USA, 3-6 November 2019; pp. 406-410.

16. Markova, E.; Moltchanov, D.; Gudkova, I.; Samouylov, K.; Koucheryavy, Y. Performance Assessment of QoS-Aware LTE Sessions Offloading onto LAA/WiFi Systems. IEEE Access 2019, 7, 36300-36311.

17. Premsankar, G.; Ghaddar, B.; Slabicki, M.; Francesco, M. Optimal configuration of LoRa networks in smart cities. IEEE Trans. Ind. Inform. 2020. [CrossRef]

18. Garbiso, J.; Diaconescu, A.; Coupechoux, M.; Leroy, B. Fair Self-Adaptive Clustering for Hybrid Cellular-Vehicular Networks. IEEE Trans. Intell. Transp. Syst. 2019, 1-12. [CrossRef]

19. Kassem, M.M.; Marina, M.K. Future wireless spectrum below 6 GHz: A UK perspective. In Proceedings of the IEEE International Symposium on Dynamic Spectrum Access Networks (DySPAN 2015), Stockholm, Sweden, 29 September-2 October 2015; pp. 59-70. [CrossRef]

20. Ojaniemi, J.; Kokkinen, H.; Kivinen, A.; Agapiou, G.; Perdikouris, S.; Hoxha, A.; Kliks, A. Coexistence of LTE Networks Under LSA Paradigm in 2.6 GHz Band. In Proceedings of the 13th EAI International Conference on Cognitive Radio Oriented Wireless Networks (CROWNCOM 2018), Ghent, Belgium, 18-20 September 2018; pp. 119-132. [CrossRef]

21. Höyhtyä, M.; Majanen, M.; Hoppari, M.; Järvensivu, P.; Kokkinen, H.; Ojaniemi, J.; Pellay, O.; Minh, D. Licensed shared access testbed for integrated satellite-terrestrial communications: The ASCENT project. In Proceedings of the 37th International Communications Satellite Systems Conference (ICSSC 2019), Naha, Japan, 29 October-1 November 2019; pp. 1-7. 
22. Lähetkangas, K.; Posti, H.; Saarnisaari, H.; Hulkkonen, A. LSA System Development with Sensing for Rapidly Deployable LTE Network. In Proceedings of the 13th EAI International Conference on Cognitive Radio Oriented Wireless Networks (CROWNCOM 2018), Ghent, Belgium, 18-20 September 2018; pp. 143-153. [CrossRef]

23. Wang, H.; Dutkiewicz, E.; Fang, G.; Mueck, M.D. Framework of joint auction and mixed graph for Licensed Shared Access systems. In Proceedings of the IEEE International Symposium on Dynamic Spectrum Access Networks (DySPAN 2015), Stockholm, Sweden, 29 September-2 October 2015; pp. 154-163. [CrossRef]

24. Wang, H.; Dutkiewicz, E.; Fang, G.; Mueck, M.D. Spectrum sharing based on truthful auction in licensed shared access systems. In Proceedings of the IEEE 82nd Vehicular Technology Conference (VTC2015-Fall), Boston, MA, USA, 6-9 September 2015; pp. 1-5. [CrossRef]

25. Raschell'a, A.; Militano, L.; Araniti, G.; Orsino, A.; Iera, A. Cognitive Management Strategies for Dynamic Spectrum Access. In Handbook of Cognitive Radio; Zhang, W., Ed.; Springer: Singapore, 2017; pp. 1-35. [CrossRef]

26. Ngo, K.; Hoppari, M.; Suomalainen, J.; Höyhtyä, M. Distributed LSA Controller for Public Safety Communications. In Proceedings of the IEEE 4th International Conference on Computer and Communications (ICCC 2018), Chengdu, China, 7-10 December 2018; pp. 1134-1138.

27. Tuukkanen, T.; Kokkinen, H.; Yrjola, S.; Ojaniemi, J.; Kivinen, A.; Jokela, T. Demonstration of Shared Spectrum Access of Different User Groups. In Proceedings of the 13th EAI International Conference on Cognitive Radio Oriented Wireless Networks (CROWNCOM 2018), Ghent, Belgium, 18-20 September 2018; pp. 36-45.

28. Yrjola, S.; Tuukkanen, T.; Kokkinen, H.; Ojaniemi, J.; Kivinen, A.; Paavola, J. Results of the Demonstration of Shared Spectrum Access of Different User Groups and Changing Priorities. In Proceedings of the Wireless Innovation Forum Conference on Wireless Communication Technologies and Software Defined Radio (WInnComm 2018), Melbourne, FL, USA, 15-16 November 2018; pp. 17-25.

29. Gudkova, I.; Samouylov, K.; Ostrikova, D.; Mokrov, E.; Ponomarenko-Timofeev, A.; Andreev, S.; Koucheryavy, Y. Service failure and interruption probability analysis for Licensed Shared Access regulatory framework. In Proceedings of the 7th International Congress on Ultra Modern Telecommunications and Control Systems and Workshops (ICUMT 2015), Brno, Czech Republic, 6-8 October 2015; pp. 123-131.

30. Gnedenko, B.; Soloviev, A. On the conditions of the existence of final probabilities for a Markov process. Math. Oper. Statist 1973, 4, 379-390.

31. Di Crescenzo, A.; Giorno, V.; Kumar, B.K.; Nobile, A. A Time-Non-Homogeneous Double-Ended Queue with Failures and Repairs and Its Continuous Approximation. Mathematics 2018, 6, 81.

32. Schwarz, J.; Selinka, G.; Stolletz, R. Performance analysis of time-dependent queueing systems: Survey and classification. Omega 2016, 63, 170-189.

33. Satin, Y.; Zeifman, A.; Sipin, A.; Ammar, S.I.; Sztrik, J. On Probability Characteristics for a Class of Queueing Models with Impatient Customers. Mathematics 2016, 8, 594.

34. Zeifman, A.; Satin, Y.; Kiseleva, K.; Korolev, V.; Panfilova, T. On limiting characteristics for a non-stationary two-processor heterogeneous system. Appl. Math. Comput. 2019, 351, 48-65.

35. Gudkova, I.; Korotysheva, A.; Zeifman, A.I.; Shilova, G.; Korolev, V.; Shorgin, S.; Razumchik, R. Modeling and analyzing licensed shared access operation for $5 \mathrm{G}$ network as an inhomogeneous queue with catastrophes. In Proceedings of the 8th International Congress on Ultra Modern Telecommunications and Control Systems (ICUMT-2016), Lisbon, Portugal, 18-20 October 2016; pp. 336-341.

36. Zeifman, A.I.; Korotysheva, A.V.; Razumchik, R.; Korolev, V.Y.; Shorgin, S.Y. Some Results for Inhomogeneous Birth-and-Death Process with Application to Staffing Problem in Telecommunication Service Systems. In Proceedings of the 7th International Congress on Ultra Modern Telecommunications and Control Systems (ICUMT-2015), Brno, Czech Republic, 6-8 October 2015; pp. 243-246.

37. Di Mauro, M.; Liotta, A. Statistical Assessment of IP Multimedia Subsystem in a Softwarized Environment: a Queueing Networks Approach. IEEE Trans. Netw. Serv. Manag. 2019, 16, 1493-1506.

38. Du, X.; Sun, Y.; Shroff, N.; Sabharwal, A. Balancing Queueing and Retransmission: Latency-Optimal Massive MIMO Design. IEEE Trans. Wirel. Commun. 2020, 19, 2293-2307. [CrossRef]

39. Rappaport, T. Wireless Communications: Principles and Practice, 2nd ed.; Prentice Hall: Upper Saddle River, NJ, USA, 2002. [CrossRef] 
40. Laya, A.; Alonso, L.; Alonso-Zarate, J.; Dohler, M. Green MTC, M2M, Internet of Things. In Green Communications: Principles, Concepts and Practice; Samdanis, K., Rost, P., Maeder, A., Meo, M., Verikoukis, C., Eds.; Wiley: Chichester, UK, 2015; pp. 217-236. [CrossRef]

41. Seo, H.; Lee, K.-D.; Yasukawa, S.; Peng, Y.; Sartori, P. LTE Evolution for Vehicle-to-Everything Services. IEEE Commun. Mag. 2016, 54, 22-28. [CrossRef]

42. ITU-R M.2370: IMT Traffic Estimates for the Years 2020 to 2030; ITU: Geneva, Switzerland, 2015.

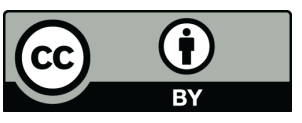

(C) 2020 by the authors. Licensee MDPI, Basel, Switzerland. This article is an open access article distributed under the terms and conditions of the Creative Commons Attribution (CC BY) license (http:/ / creativecommons.org/licenses/by/4.0/). 